\title{
Current-density-functional approach to large quantum dots in intense magnetic fields
}

\author{
M. Pi, M. Barranco, A. Emperador, and E. Lipparini* \\ Departament d'Estructura i Constituents de la Matèria, Facultat de Física, Universitat de Barcelona, E-08028 Barcelona, Spain \\ L1. Serra \\ Departament de Física, Facultat de Ciències, Universitat de les Illes Balears, E-07071 Palma de Mallorca, Spain
}

(Received 13 November 1997)

\begin{abstract}
Within current-density-functional theory, we have studied a quantum dot made of 210 electrons confined in a disk geometry. The ground state of this large dot exhibits some features as a function of the magnetic field $(B)$ that can be attributed in a clear way to the formation of compressible and incompressible states of the system. The orbital and spin angular momenta, the total energy, ionization and electron chemical potentials of the ground state, as well as the frequencies of far-infrared edge modes are calculated as a function of $B$, and compared with available experimental and theoretical results.
\end{abstract}

[S0163-1829(98)01923-7]

\section{INTRODUCTION}

The study of the formation of compressible and incompressible states of quantum dots has attracted enormous interest in the last few years. It has been argued ${ }^{1-4}$ that for a sufficiently smooth confining potential, the edge of the dot exhibits to a certain degree narrow incompressible regions where the electronic density is determined by the filling factor $\nu$ as $\rho=\nu /\left(2 \pi \mathcal{L}^{2}\right)$, where $\mathcal{L}=(\hbar c / e B)^{1 / 2}$ is the magnetic length, and compressible regions where $\rho$ may vary with the radial distance $r$. These states are at the origin of phenomena such as, e.g., "magic" angular momentum quantum numbers, composite droplets and edge reconstruction. ${ }^{5,6}$ Some evidence for the formation of these states has been found in the magnetic field $(B)$ dependence of the addition energies, i.e., the energies to add one more electron to the $\operatorname{dot}^{7,8}$ and more recently, in the oscillations of the resonance frequencies of edge magnetoplasmon modes of dots and antidots. ${ }^{9}$

Exact diagonalizations of the electronic Hamiltonian corresponding to dots with a small number of electrons $N$ ( $N$ $\leqslant 8$ ) (Refs. 10-13) have shown that these systems have strongly correlated ground states (g.s.), especially at high magnetic fields. Rigorous results have been obtained in the high-density limit; see Ref. 14. Approximate schemes such as the constant interaction model of Ref. 15, the selfconsistent model of Ref. 16, the Hartree-Fock method of Refs. 6 and 17, or other mean field approximations ${ }^{18}$ which have been used to carry out calculations in larger dots, do not take into account these correlations, so they may fail in reproducing a number of g.s. features that appear as a function of $B \cdot{ }^{13}$ It is worth mentioning that all these approaches have been only applied to the description of medium size ( $N$ $<100$ ) dots and relatively small values of $B$ due to the technical difficulties one has to face otherwise because of the high density of single particle (s.p.) states.

Recently, current density-functional theory ${ }^{19}$ (CDFT) has been used to study g.s. properties of small quantum dots. ${ }^{20}$ The accuracy of the method has been tested in the case of two and three electron systems, for which exact calculations are available. ${ }^{11}$ It has been shown ${ }^{20}$ that CDFT reproduces accurately the exact results, thus providing convincing evidence that it is a powerful tool to quantitatively describe the g.s. properties of quantum dots in magnetic fields. CDFT has also been used to study far-infrared edge magnetoplasmon modes in medium size quantum dots by means of an equation of motion method. ${ }^{21}$

In the present work, we extend the CDFT calculations to the case of a large dot of radius $R=1600 \AA$ containing 210 electrons in a magnetic field ranging from $B=0$ to $\sim 15 \mathrm{~T}$. The far-infrared spectrum of this dot has been experimentally studied in Ref. 22. The large number of electrons makes this dot closer to the two-dimensional (2D) conditions than any other system previously considered in a calculation of this kind. Moreover, the high $B$ 's here attained allow us to study g.s.'s that are associated in a clear way with bulk incompressible states with filling factors $\nu=\frac{2}{3}$, and from $\nu=1$ to 10 , and to several incompressible states in between. Such a rich structure has not been previously disclosed in a single large dot, where genuine magnetic effects, even at rather small $B$, are not masked by surface nor finite-size effects.

The density functional we are using does not incorporate one of the key features to describe the occurrence in a single dot of several incompressible strip regions in the fractional quantum Hall regime, namely, cusps in the exchangecorrelation scalar potential at certain filling factors (see, for example, Refs. 4 and 23). However, it is able to describe the appearance of bulk regions with a fractional filling factor. This is illustrated in the case of the $\nu=\frac{2}{3}$ incompressible state.

To fully display these magnetic field effects, we have performed the calculations at a very small temperature $(T), 0.1$ $\mathrm{K}$. The use of such temperatures in the calculations is a challenge for large dots and intense magnetic fields. Some results at higher $T$ are also discussed. Finally, we have obtained the far-infrared spectrum of the dot.

\section{CURRENT-DENSITY-FUNCTIONAL DESCRIPTION OF QUANTUM DOTS}

Current-density-functional theory is comprehensively described in Ref. 19 and has been already applied to quantum 
dots. $^{20,21}$ In the present work, we shall be considering $N$ electrons moving in the $z=0$ plane where they are confined by the dot potential $V_{+}(r)$ with $r=\sqrt{x^{2}+y^{2}}$. On this system it may act a constant magnetic field in the $z$ direction described by the vector potential $\vec{A}=\frac{1}{2}(-y, x, 0) B$. Introducing the cyclotron frequency $\omega_{c}=e B / m c$, we can write the CDFT total grand potential $\mathcal{A}=E-T S-\mu N$ adding to the total energy

$$
\begin{aligned}
E= & \frac{1}{2} \int d \vec{r} \tau(\vec{r})+\frac{\omega_{c}}{2} \int d \vec{r} r j_{p}(\vec{r})+\frac{1}{8} \omega_{c}^{2} \int d \vec{r} r^{2} \rho(\vec{r}) \\
& +g^{*} \mu_{B} B \sum_{i} f_{i^{\prime}} s_{z_{i}}+\int d \vec{r} V_{+}(\vec{r}) \rho(\vec{r}) \\
& +\frac{1}{2} \iint d \vec{r} d \vec{r}^{\prime} \frac{\rho(\vec{r}) \rho\left(\vec{r}^{\prime}\right)}{\left|\vec{r}-\vec{r}^{\prime}\right|}+E_{x c}(\rho, \xi, \overrightarrow{\mathcal{V}}),
\end{aligned}
$$

the temperature times the total entropy $S$ given by

$$
S=-\sum_{i}\left[f_{i} \ln f_{i}+\left(1-f_{i}\right) \ln \left(1-f_{i}\right)\right]
$$

and the electronic chemical potential $\mu$ times the electron number $N=\sum_{i} f_{i}$.

To simplify the formulas, we have used effective atomic units, defined by $\hbar=e^{2} / \epsilon=m=k_{B}=1$, where $k_{B}$ is the Boltzmann's constant and $m=m^{*} m_{e}$ is the electron effective mass. In this system of units, the length unit is the Bohr radius $a_{0}$ times $\epsilon / m^{*}$, and the energy unit is the Hartree times $m^{*} / \epsilon^{2}$, which we call, respectively, $a_{0}^{*}$ and $E_{H}^{*} \cdot m^{*}$ is the electron effective mass in units of the bare one, which together with a dielectric constant $\epsilon$ and a gyromagnetic factor $g^{*}$ are characteristics of the semiconductor (for example, $g^{*}=-0.44, \epsilon=12.4$, and $m^{*}=0.067$ in GaAs), and $\mu_{B}$ $=\hbar e /\left(2 m_{e} c\right)$ is the Bohr magneton. ${ }^{24}$ For GaAs we have $a_{0}^{*} \sim 97.94 \AA$ and $E_{H}^{*} \sim 11.86 \mathrm{meV}$.

In Eqs. (1) and (2), $s_{z_{i}}$ is the $z$ component of the spin, and $f_{i}$ is the occupation number of the $i$-s.p. level. The particle $\rho(\vec{r})$, kinetic energy $\tau(\vec{r})$, and paramagnetic current densities $\vec{j}_{p}(\vec{r})$ are defined in terms of the occupation numbers and s.p. wave functions $\phi_{i}$ as follows:

$$
\begin{gathered}
\rho(r)=\sum_{i} f_{i}\left|\phi_{i}(r)\right|^{2}, \\
\tau(r)=\sum_{i} f_{i}\left|\nabla \phi_{i}(r)\right|^{2}, \\
\vec{j}_{p}(r)=j_{p}(r) \hat{e}_{\theta}=-\frac{1}{r} \sum_{i} f_{i} l_{i}\left|\phi_{i}(r)\right|^{2} \hat{e}_{\theta},
\end{gathered}
$$

where $\hat{e}_{\theta}$ is the azimuthal unit vector. Due to the axial symmetry, the electronic wave functions can be factorized as

$$
\phi(\vec{r})=e^{-\imath l \theta} u_{n l \sigma}(r)
$$

where $\sigma= \pm 1, n=1,2,3 \ldots$, and $l=0, \pm 1, \pm 2, \pm 3 \ldots$. These wave functions are eigenstates of the angular momentum around the $z$ axis with eigenvalue $-l$. At $B \neq 0$, the s.p. level $i$ is nondegenerate, and in the above expressions we have used the short notation $i \equiv\{n, l, \sigma\} . V_{+}(r)$ is the Coulomb potential generated by a jellium disk of radius $R$ and areal density $\rho_{+}=\rho_{b} \Theta(R-r),{ }^{25}$ and the $\rho_{b}$ value has been fixed by charge neutrality, i.e., $\pi \rho_{b} R^{2}=N_{+}=N$. For the $R$ $=1600 \AA$ and $N=210$ system that corresponds to one of the GaAs dots studied in Ref. 22, one has $\rho_{b}=0.239 a_{0}^{*-2}$. The use of a step function to model the positive neutralizing background, i.e., a sharp confining potential, ${ }^{26}$ together with a smooth exchange-correlation potential, implies that no strips of filling factor smaller than the one at the center of the droplet are expected to appear at the edge of the dot. That will be confirmed by the calculations presented in the next section

The exchange-correlation energy $E_{x c}(\rho, \xi, \overrightarrow{\mathcal{V}})$ in Eq. (1) has been taken from Ref. 20. It is a functional of the particle density $\rho$, the local spin polarization $\xi$,

$$
\xi(\vec{r})=\frac{\rho_{\uparrow}-\rho_{\downarrow}}{\rho_{\uparrow}+\rho_{\downarrow}},
$$

where $\rho_{\uparrow}\left(\rho_{\downarrow}\right)$ is the particle density of spin up(down) electrons, and of the local vorticity $\overrightarrow{\mathcal{V}}(r)$ :

$$
\overrightarrow{\mathcal{V}}(r)=\mathcal{V}(r) \hat{e}_{z}=-\frac{c}{e r} \frac{\partial}{\partial r}\left(r \frac{j_{p}}{\rho}\right) \hat{e}_{z} .
$$

For further reference, we also define the local spin magnetization $m(r)$ as $m(r)=\rho(r) \xi(r)$.

To obtain the s.p. wave functions $\phi_{i}(\vec{r})$ and occupation numbers $f_{i}$, one has to minimize $\widetilde{\mathcal{A}}=\mathcal{A}-\Sigma_{i} \lambda_{i}\left\langle\phi_{i} \mid \phi_{i}\right\rangle$. Minimizing $\mathcal{A}$ with respect to the wave functions one obtains the following Kohn-Sham (KS) equations:

$$
\begin{gathered}
{\left[-\frac{1}{2}\left(\frac{\partial^{2}}{\partial r^{2}}+\frac{1}{r} \frac{\partial}{\partial r}-\frac{l^{2}}{r^{2}}\right)-\frac{\omega_{c}}{2} l+\frac{1}{8} \omega_{c}^{2} r^{2}+V_{+}(r)\right.} \\
\quad+\int d \vec{r}^{\prime} \frac{\rho\left(\vec{r}^{\prime}\right)}{\left|\vec{r}-\vec{r}^{\prime}\right|}-\frac{e}{c} l \frac{A_{x c}(r)}{r}+V_{x c \sigma}(r) \\
\left.+\frac{1}{2} g^{*} \mu_{B} B \sigma\right] u_{n l \sigma}=\epsilon_{n l \sigma} u_{n l \sigma},
\end{gathered}
$$

with $\epsilon_{i} \equiv \lambda_{i} / f_{i}$ and where

$$
V_{x c \sigma}(r)=\left.\frac{\delta E_{x c}(\rho, \xi, \overrightarrow{\mathcal{V}})}{\delta \rho_{\sigma}}\right|_{\rho_{-\sigma}, \overrightarrow{\mathcal{V}}}-\frac{e}{c} \vec{A}_{x c}(r) \cdot \frac{\vec{j}_{p}}{\rho},
$$

where $\vec{A}_{x c}$ is the exchange-correlation vector potential

$$
\frac{e}{c} \vec{A}_{x c}(r)=\frac{e}{c} A_{x c} \hat{e}_{\theta}=\frac{c}{e \rho} \frac{\partial}{\partial r}\left(\left.\frac{\delta E_{x c}}{\delta \mathcal{V}}\right|_{\rho, \xi}\right) \hat{e}_{\theta} .
$$

Minimizing $\widetilde{\mathcal{A}}$ with respect to $f_{i}$ and making use of the KS equations, one obtains the occupation numbers

$$
f_{i}=\frac{1}{1+e^{\left(\epsilon_{i}-\mu\right) / T}} .
$$



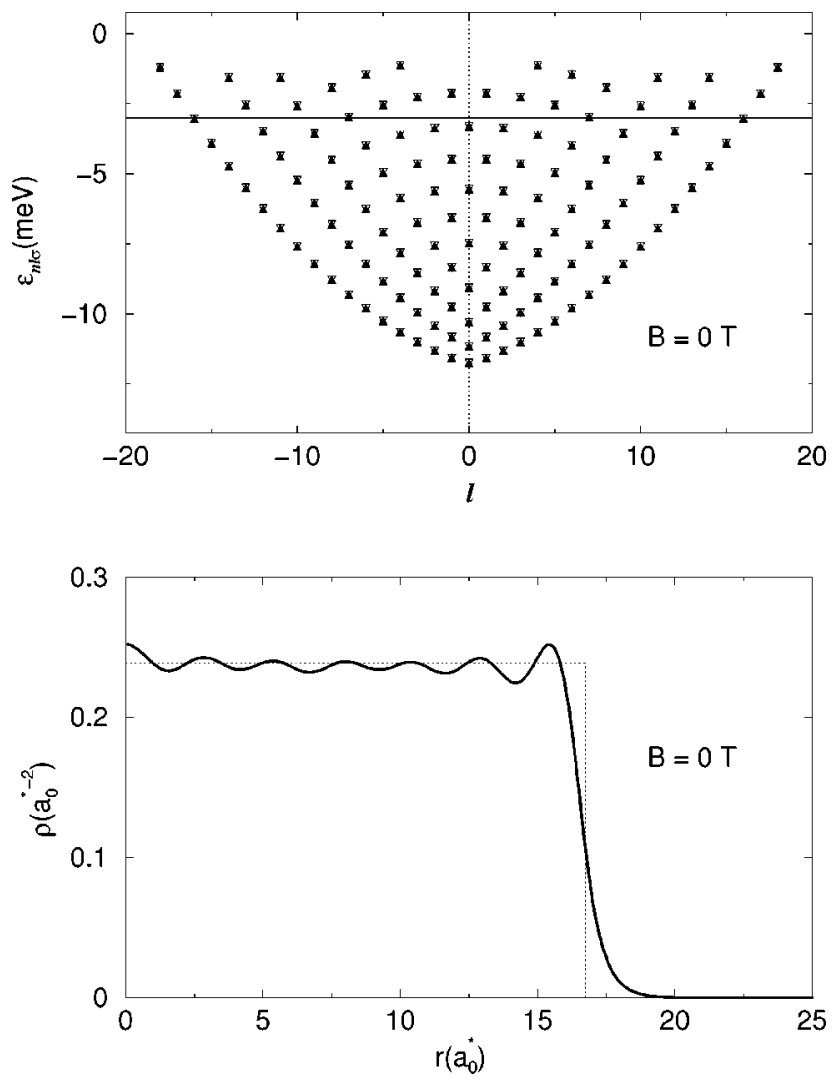

FIG. 1. Top panel: s.p. energies as a function of orbital angular momentum $l$ at $B=0$. The full, upright triangles represent spin-up states, and the empty, downright triangles, spin-down states. The horizontal solid line represents the electron chemical potential. Bottom panel: the corresponding electron density as a function of $r$, both in effective atomic units. The dotted line represents the jellium density.

The electron chemical potential is fixed from the normalization condition

$$
N=\sum_{i} \frac{1}{1+e^{\left(\epsilon_{i}-\mu\right) / T}} .
$$

The KS differential equations and the normalization condition Eq. (13) have been solved self-consistently, without expanding the s.p. wave functions in a necessarily truncated basis of Fock-Darwin or Landau orbitals. Our iterative method works for weak and strong $B$ fields as well, for which the effective potential is very different. It has the advantage of avoiding the study of how the results depend on the size of the basis.

\section{GROUND-STATE RESULTS}

We have studied the g.s. of the $N=210$ electrons dot at $T=0.1 \mathrm{~K}$ varying the magnetic field from 0 to $\sim 15.5 \mathrm{~T}$ in order to explore states with filling factors $\nu=\frac{2}{3}$ and above. We recall that integer $\nu$ 's also represent the number of occupied Landau bands, which we label as $(M, \uparrow)$ or $(M, \downarrow)$, depending on the spin of the s.p. states in the band. $M=n$ $+(|l|-l) / 2$ is the Landau-level index.

The density and single-particle energies corresponding to the $B=0$ case are represented in Fig. 1. The s.p. energies are
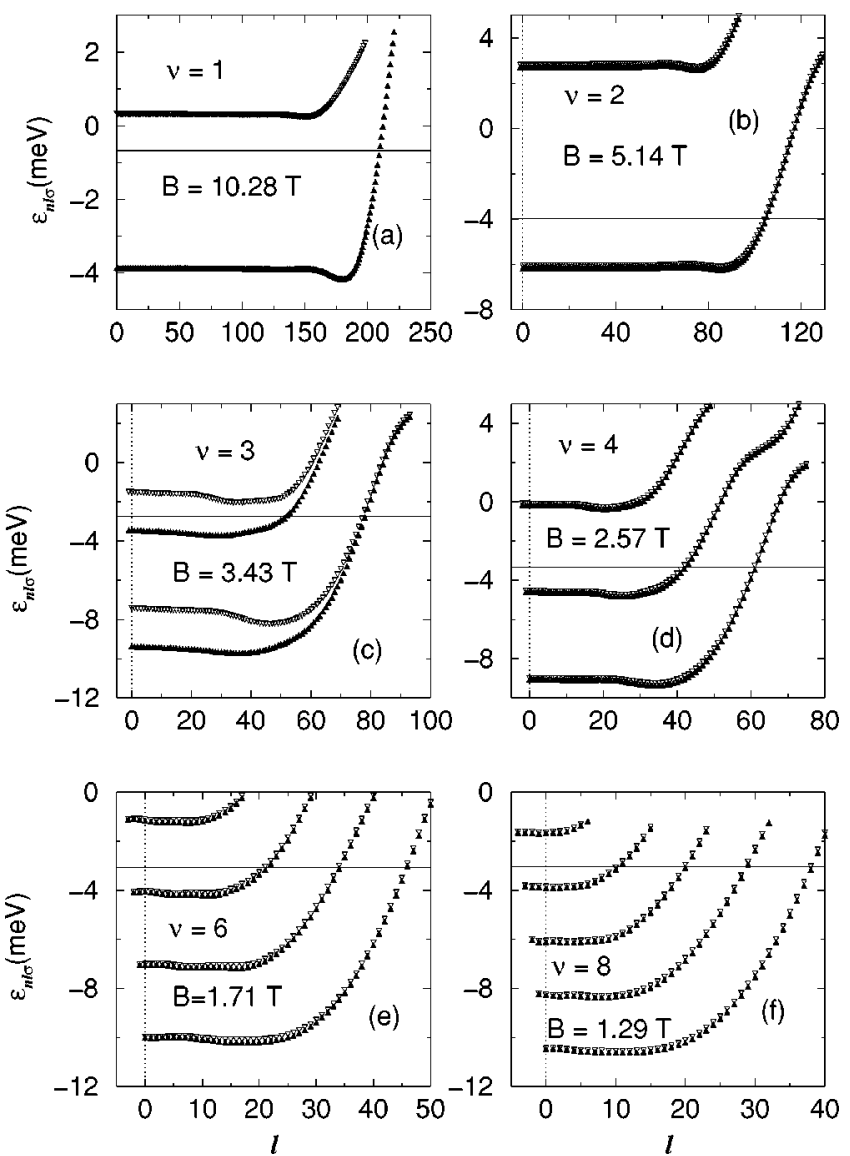

FIG. 2. Single-particle energies as a function of orbital angular momentum $l$ for $\nu=1,2,3,4,6$, and 8. The horizontal solid lines represent the electron chemical potential. The full, upright triangles represent $(M, \uparrow)$ bands, and the empty, downright triangles, $(M, \downarrow)$ bands. In panel (a), only $M=1$ bands appear, whereas in panel (f), bands with $M=1$ to 5 are visible.

distributed along paraboliclike curves as a function of $l$, each curve being characterized by a different value of $n$. The figure shows the well-known fourfold degeneracy of each single-particle level, corresponding to the possibilities $\pm l$, $(\uparrow \downarrow)$. It is remarkable that even for such a large system, the density still displays many oscillations in the bulk that have to be gradually washed out as the dot evolves towards the $2 \mathrm{D}$ system.

Figure 2 shows the CDFT s.p. energy spectra for $B$ $=10.28,5.14,3.43,2.57,1.71$, and $1.29 \mathrm{~T}$ as a function of $n$ and $l$. Observe that due to the sign of the linear $l$ term in Eq. (9), most occupied levels have now a positive $l$ value, i.e., a negative orbital angular momentum, and that the $(M, \uparrow)$ band lies below the $(M, \downarrow)$ one because of the negative value of $g^{*}$. Figure 3 shows the corresponding g.s. particle $\rho(r)$ and local spin magnetization $m(r)$ densities together with the predicted values of total orbital and spin angular momentum $\left(L_{z}, 2 S_{z}\right)$.

From these figures, one can see that these states are the finite-size analog of the 2D Landau incompressible states with $\nu=1-4,6$, and 8, which, for an electronic density $\rho_{e}$ $=\rho_{b}=0.239 a_{0}^{*-2}$, would be precisely realized at the above $B$ values. Indeed, the densities in Fig. 3 exhibit a steplike shape whose plateau has a density determined by an integer filling factor $\nu$ as $\rho=\nu e B / 2 \pi c$. To help follow the discus- 

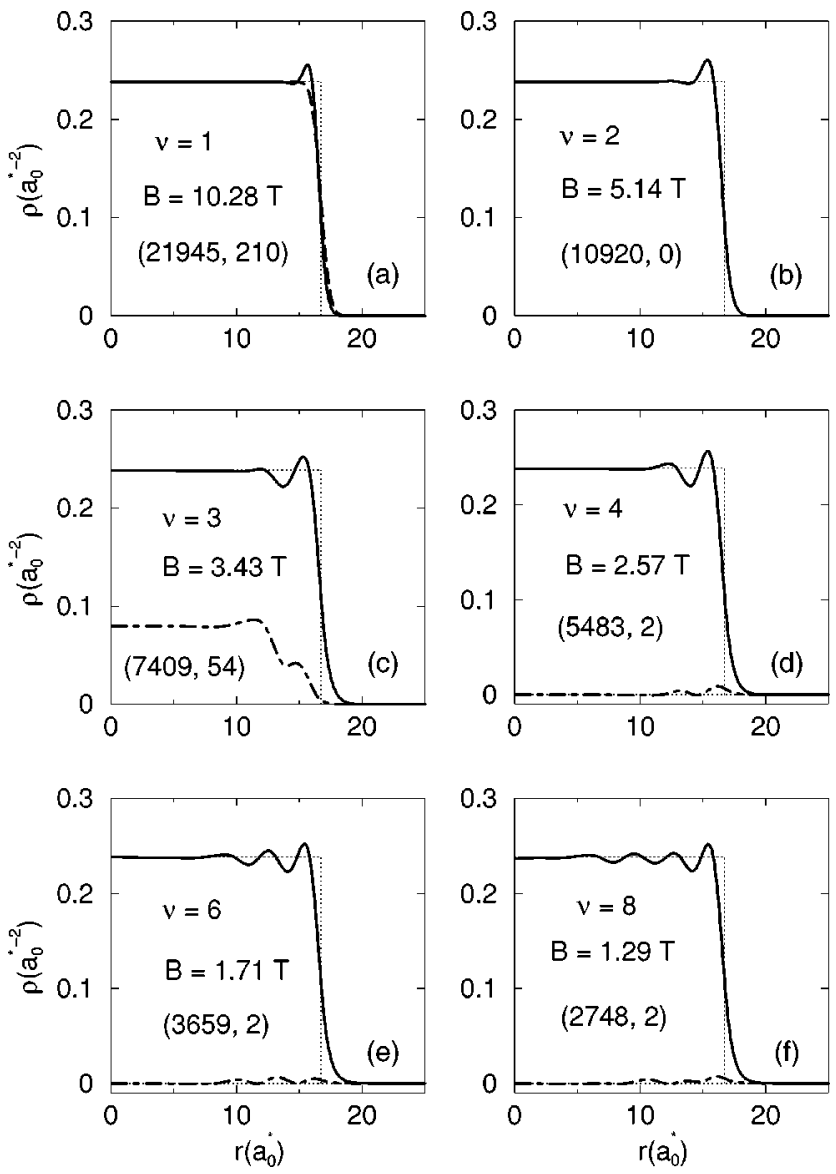

FIG. 3. Electron $\rho(r)$ (solid lines) and local spin magnetization $m(r)$ (dot-dashed lines) densities corresponding to the configurations displayed in Fig. 2. The dashed curve in panel (a) represents the density of the MDD state. All densities are in effective atomic units. Also shown are the values of $\left(L_{z}, 2 S_{z}\right)$.

sion of the results we present, we have collected in Table I the values of $B$ and $\mathcal{L}$ that correspond to $\nu=1$ to 10 . Notice that even for $\nu=10, R$ is much larger than $\mathcal{L}$, so that the filling factor $\nu$ can be safely used to describe the occupation of the Landau bands in the interior of the droplet.

The g.s. values of the orbital angular momentum agree well with the law

$$
L_{z}=\nu^{-1} \frac{N(N-1)}{2},
$$

TABLE I. Values of $B$ and $\mathcal{L}$ corresponding to integer filling factors $\nu=1$ to 10 .

\begin{tabular}{lcc}
\hline \hline$\nu$ & $B(\mathrm{~T})$ & $\mathcal{L}\left(a_{0}^{*}\right)$ \\
\hline 1 & 10.28 & 0.82 \\
2 & 5.14 & 1.15 \\
3 & 3.43 & 1.41 \\
4 & 2.57 & 1.63 \\
5 & 2.06 & 1.82 \\
6 & 1.71 & 2.00 \\
7 & 1.47 & 2.16 \\
8 & 1.29 & 2.31 \\
9 & 1.14 & 2.45 \\
10 & 1.03 & 2.58 \\
\hline \hline
\end{tabular}

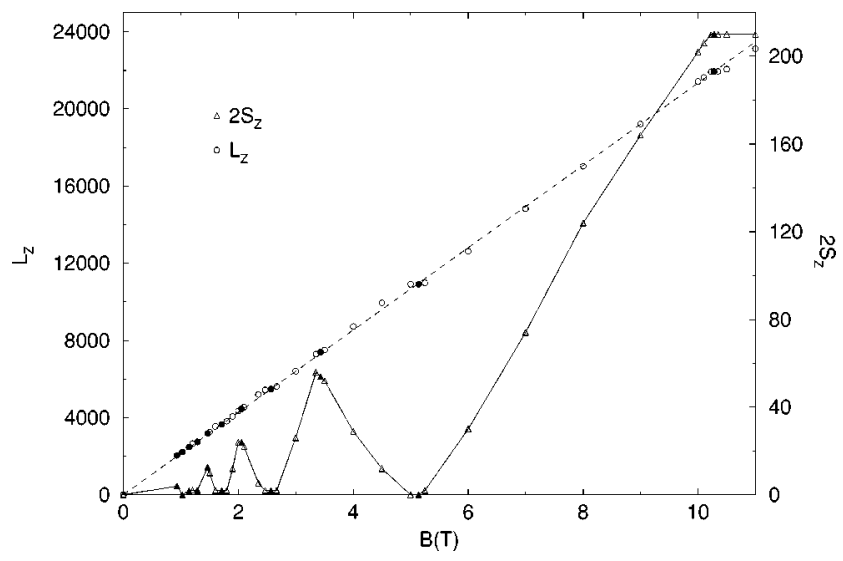

FIG. 4. $L_{z}$ (circles, left scale) and $2 S_{z}$ (triangles, right scale) as a function of $B$. The values corresponding to integer filling factors 1 to 11 are denoted by solid symbols, and the line connecting the $2 S_{z}$ points is to guide the eye. The dashed line represents Eq. (14).

and the spin is approximately given by

$$
2 S_{z}=\left\{\begin{array}{lll}
0, & \nu & \text { even }, \\
\frac{N}{\nu}, & \nu & \text { odd },
\end{array}\right.
$$

expressions that are valid in the big- $N$ limit for the Landau bands. This is explicitly shown in Fig. 4, where we have plotted the calculated orbital and spin angular momenta as a function of $B$. The small plateaus along the $L_{z}(B)$ curve at integer $\nu$, also visible in the $2 S_{z}(B)$ curve, show the robustness of these states against changes in $B$, and their tendency to not to change their $\left(L_{z}, S_{z}\right)$ values.

In panel (a) of Fig. 3 we have plotted the density of the maximum density droplet (MDD) state: ${ }^{27}$

$$
\rho_{\mathrm{MDD}}(r)=\frac{1}{2 \pi \mathcal{L}^{2}} \sum_{l=0}^{N-1} \frac{1}{l !}\left(\frac{r}{\sqrt{2} \mathcal{L}}\right)^{2 l} e^{-r^{2} / 2 \mathcal{L}^{2}}
$$

One can see that both densities are very similar, the only difference appears in the edge region. The lump in the CDFT density at the edge is due to the rather sharp confining potential, on the one hand, and on the other hand to the exchange-correlation energy, which is changing rapidly in the surface region of the droplet, see Eqs. (10) and (11), and Ref. 23.

It is also interesting to look at the energy difference between the $(M, \uparrow)$ and $(M, \downarrow)$ Landau bands shown, for example, in Fig. 2. It is very small for even $\nu$ 's, which correspond to g.s.'s having $S_{z} \simeq 0$, because the Zeeman term in Eq. (1) is also small $(0.127 \mathrm{meV}$ at $B=5 \mathrm{~T}$, for instance). On the contrary, that separation is sizable for the $\nu=1$ and 3 cases, in the 2-4-meV range. These g.s. states have $S_{z} \neq 0$. One should have in mind that $\omega_{c}$, which gives the difference of frequencies between Landau levels when spin and interaction effects are neglected, is around $18 \mathrm{meV}$ for $B=10 \mathrm{~T}$. $V_{x c \sigma}$ is the origin of the large spin splittings. The importance of the exchange energy in the spin splitting of the Landau bands was already pointed out in Ref. 17 . The similarity of the g.s. corresponding to $\nu=1$ and the MDD state is due to 

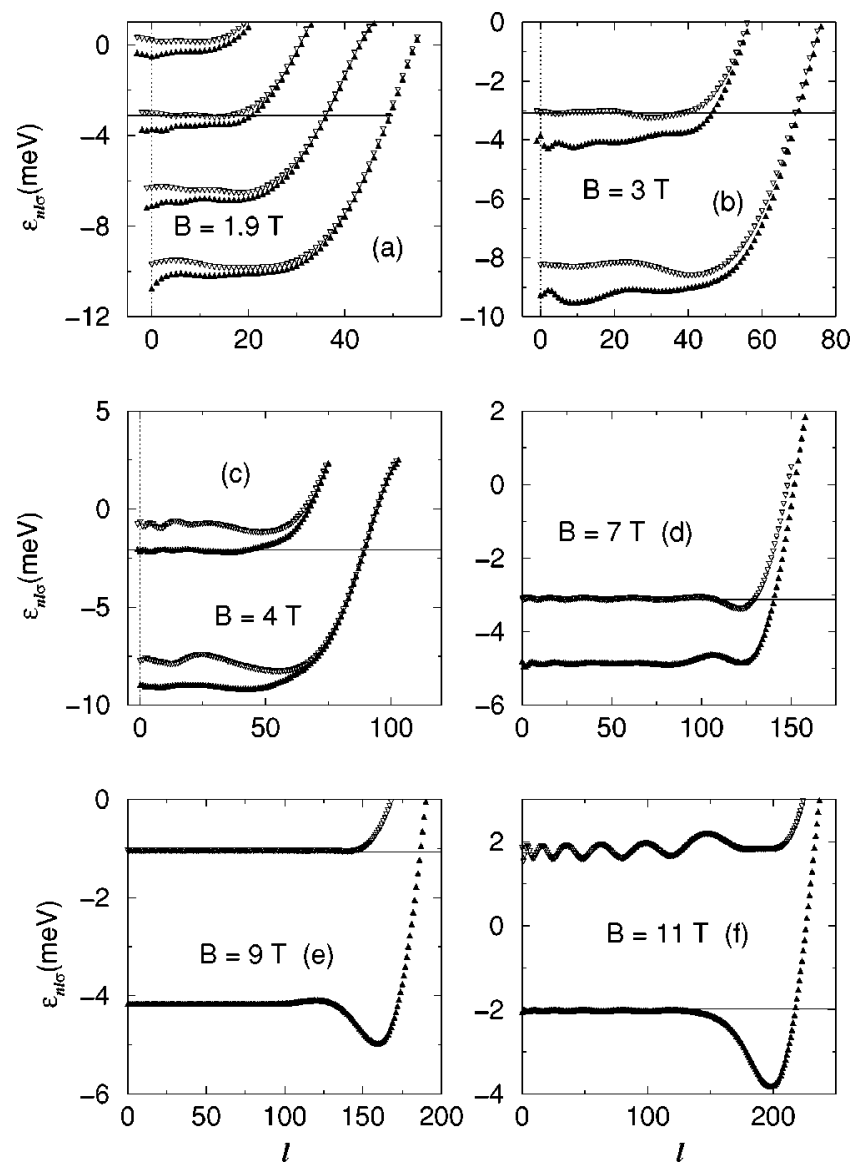

FIG. 5. Single particle energies as a function of orbital angular momentum $l$ for $B=1.9,3,4,7,9$, and $11 \mathrm{~T}$, respectively. The horizontal solid lines represent the electron chemical potential. Same notation as in Fig. 2.

$V_{x c \sigma}$, as it prevents the $(1, \uparrow)$ and $(1, \downarrow)$ Landau bands from being close at $B=10.28 \mathrm{~T}$ and, thus, to contribute both to the building of the g.s.

We have not attempted to disentangle which part of the splitting we have found is due to the exchange energy, and which part is due to the electronic correlations. It would be possible at low $B$ as the exchange-correlation energy comes from the Tanatar-Ceperly parametrization, ${ }^{28}$ but not at high $B$ because of the form of the parametrization given by Levesque et al. ${ }^{29}$ Exchange-correlation effects enhanced by the finite size of the system are also responsible for the differences between the calculated values of $L_{z}$, and especially of $S_{z}$, and the ones given by Eqs. (14) and (15). In the case of $S_{z}$, these differences can be sizable for "large" odd filling factors; see Fig. 4.

Figures 5 and 6 show the s.p. energies and densities for $B=1.9,3,4,7,9$, and $11 \mathrm{~T}$. These values originate compressible g.s.'s in the dot, and a careful look at these figures sheds light on the appearance of compressible and incompressible states in the finite system.

When the magnetic field is increased from that corresponding to $\nu=1$, some electrons are promoted within the $(1, \uparrow)$ band to s.p. states with higher $l$ values. This is seen in panel (f) of Fig. 5. Indeed, it can be realized from that figure that the chemical potential intersects the s.p. curve at a value of $l$ larger than 210, and that at the same time, some low- $l$
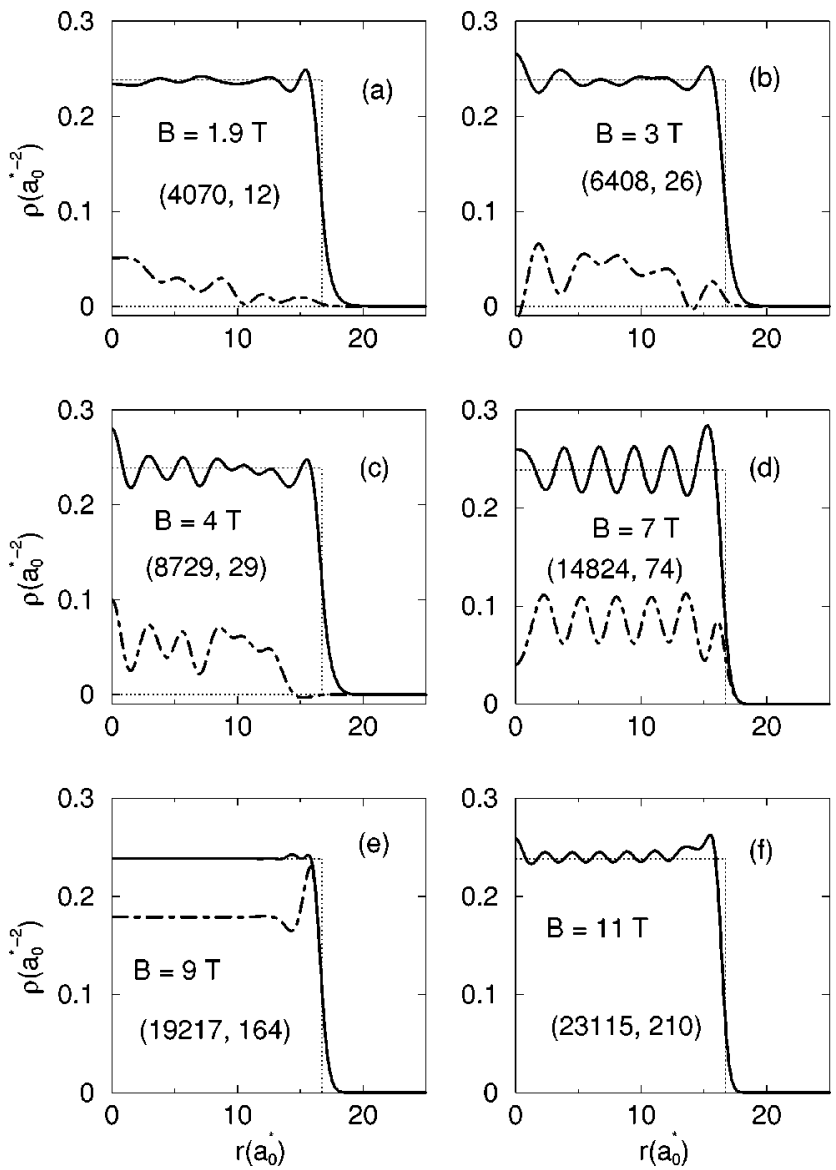

FIG. 6. Electron $\rho(r)$ (solid lines) and local spin magnetization $m(r)$ (dot-dashed lines) densities in effective atomic units corresponding to the configurations displayed in Fig. 5. Also shown are the values of $\left(L_{z}, 2 S_{z}\right)$.

s.p. levels are at an energy slightly above $\mu$. This is precisely the evolution of the MDD state when $B$ is increased and edge reconstruction occurs. ${ }^{30,31}$

A different behavior is shown in the other panels of Fig. 5. Consider, for instance, the situation displayed in panel (d). Around 140 electrons are in the $(1, \uparrow)$ band, as may be inferred from the intersection point of that band with the $\mu$ line. All other electrons are oddly distributed in the $(1, \downarrow)$ band, starting from the s.p. levels with higher orbital angular momentum, whose energies are well below the chemical potential line because of the dip around $l=120$ caused by the exchange-correlation energy term. Whenever the partially filled Landau band presents a flat region, the electron chemical potential has to be the average s.p. energy of that region. Otherwise, electrons will continue to fill s.p. levels with high- $l$ values belonging to the previous band.

The corresponding electron density manifests the peculiarities of the s.p. spectrum in quite a distinct manner: the oscillations displayed by the density at $B=7 \mathrm{~T}$ as shown in panel (d) of Fig. 5 are washed out at $B=9 \mathrm{~T}$, as shown in panel (e) of the same figure. This is because for this magnetic field the distribution of s.p. energies of the $(1, \downarrow)$ band evens out around $\mu$.

The evolution of the compressible region between $\nu=2$ and 1 when $B$ increases from 5.14 to $10.28 \mathrm{~T}$ deserves further discussion, as this region extends in a wide $B$ range and 
it makes the magnetic effects easier to visualize (a similar evolution has been found between $\nu=3$ and 2). After a regime extending up to $\geqslant 7 \mathrm{~T}$ in which the droplet densities present an oscillatory behavior, at $B \sim 8 \mathrm{~T}$ the $(1, \downarrow)$ flattens out and accordingly, the density oscillations disappear. The configuration at $B=8 \mathrm{~T}$ having $L_{z}=17031$ and $2 S_{z}=124$ cannot be identified with the $\nu=1$ incompressible state, since $L_{z}$ and $2 S_{z}$ are far from having the appropriated values, or other similar g.s. configurations corresponding to magnetic fields as high as $10 \mathrm{~T}$, see Fig. 4. This means that looking only at the densities might be misleading in identifying integer filling factor states. The evolution of the $B$ $=10 \mathrm{~T}$ state towards the $\nu=1$ one at $10.28 \mathrm{~T}$ proceeds through an interesting change in the s.p. spectra of the $(1, \downarrow)$ band: it bends upwards crossing the $\mu$ line, so that its occupied higher- $l$ states become now empty. To keep constant the number of electrons on the one hand, and to keep $L_{z}$ increasing with $B$ on the other hand, the $(1, \uparrow)$ band extends its occupied s.p. states up to higher- $l$ states. A further increase in $B$ "decouples" $\mu$ from the $(1, \downarrow)$ band, and the situation is eventually that shown in panel (a) of Fig. 2.

It is of some importance to figure out what is the quantitative influence of temperature on the above features of the g.s. To this end, we have carried out calculations using a moderate $T$, namely, $2 \mathrm{~K}$. For quantum dots as the one studied here, it appears that the density of states around the chemical potential is large, and such a small temperature already produces changes in the s.p. spectrum and the electron density as well. This is illustrated in Fig. 7, where we have drawn the s.p. energies and electron densities corresponding to $B=4$ and $7 \mathrm{~T}$.

A comparison of cold and hot s.p. energies in Fig. 7 shows that a first thermal effect is that the small wiggles around $\mu$ in the cold spectrum are progressively wiped out when $T$ increases. In other words, the low- $l$ states, which are unoccupied at $T=0.1 \mathrm{~K}$ (they lay slightly above the chemical potential), are now occupied with some probability. This flattens the electron density in the bulk of the droplet. A second thermal effect is the deepening of the high- $l$ s.p. levels as $T$ increases. This is due to the widening of the surface region, a situation that has also been met in other finite fermionic systems; see, for example, Refs. 32-34. This thermal effect reduces the amplitude of the density oscillation at the edge of the dot.

It is thus quite apparent that to study the incompressible states, one has to use temperatures in the right range of values. Otherwise, the thermal average implicit in the definition of one-body densities will wash out the quantum oscillations of compressible states, making the identification of the incompressible ones more difficult.

At $B=15.42 \mathrm{~T}$, an incompressible state appears, which deserves a separate analysis. From the relationship $\rho$ $=\nu e B / 2 \pi c$, it would correspond to a filling factor $\nu=\frac{2}{3}$. We predict for this state an angular momentum $L_{z}=32511$, which is very close to the one given by Eq. (14), namely, 32 917. We recall that the angular momentum of a droplet in a Laughlin's state ${ }^{35}$ is given by that equation only when $\nu^{-1}$ equals $1,3,5, \ldots$. We argue that this formula is a good approximation not only for integer filling factors and those of
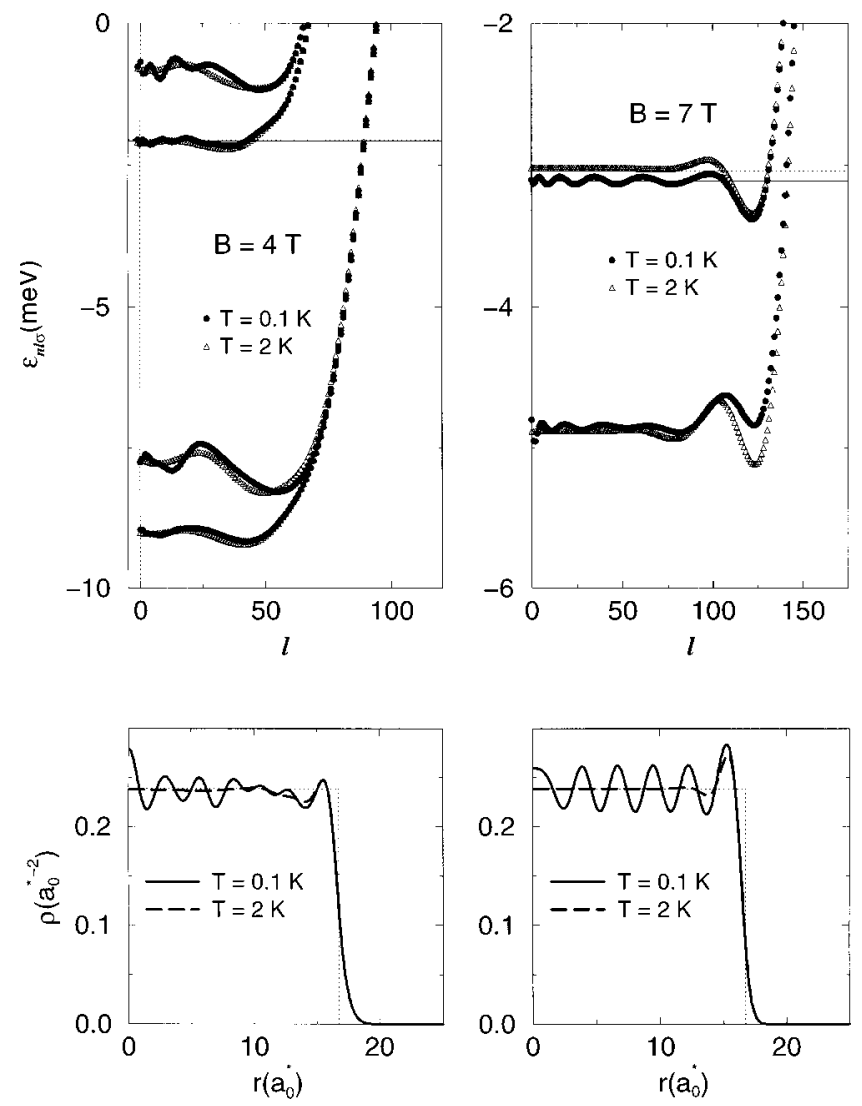

FIG. 7. Top panels: s.p. energies at $T=0.1 \mathrm{~K}$ (dots), and at $T$ $=2 \mathrm{~K}$ (triangles). Bottom panels: Electron density $\rho(r)$ at $T=0.1 \mathrm{~K}$ (solid line), and at $T=2 \mathrm{~K}$ (dashed line). The left panels correspond to $B=4 \mathrm{~T}$, and the right ones to $B=7 \mathrm{~T}$. The electron chemical potential is indicated by a horizontal line (solid, $T=0.1 \mathrm{~K}$; dashed, $T=2 \mathrm{~K})$.

Laughlin's states, but also for $\nu=\frac{2}{3}$. As a matter of fact, Fig. 4 shows that Eq. (14) works well even for compressible states.

As we show in panel (c) of Fig. 8, the electron density is constant in the bulk and has a bump at the dot edge. This has also been found in simulation calculations of Laughlin's states. ${ }^{36,37}$ However, these simulations have been carried out for few electron dots, and density oscillations are still visible in the bulk. In the CDFT calculation presented here, the density is constant in the bulk, which, together with the associated values of $L_{z}$ and $2 S_{z}$, makes it clear to label this dot state with a filling factor $\nu=\frac{2}{3}$.

Further insight into this state can be obtained splitting its density as follows:

$$
\begin{aligned}
\rho_{\frac{2}{3}}(r) & =\sum_{i=0}^{N-1} f_{i}\left|\phi_{i}(r)\right|^{2}+\sum_{i \geqslant N} f_{i}\left|\phi_{i}(r)\right|^{2} \\
& \equiv \rho_{\text {bulk }}(r)+\rho_{\text {surf }}(r),
\end{aligned}
$$

with $i \equiv\{1, l, \uparrow\}$. This produces the result displayed in panel (c) of Fig. 8, where $\rho_{\text {bulk }}(r)$ is represented by a dashed line, and $\rho_{\text {surf }}(r)$ by a dot-dashed line. The integral over $r$ of the former gives $N_{\text {bulk }} \simeq 140$, and that of the latter, $N_{\text {surf }} \simeq 70$. This means that two-thirds of the electrons in the dot are in the bulk region, and one third in the edge region. A look at 

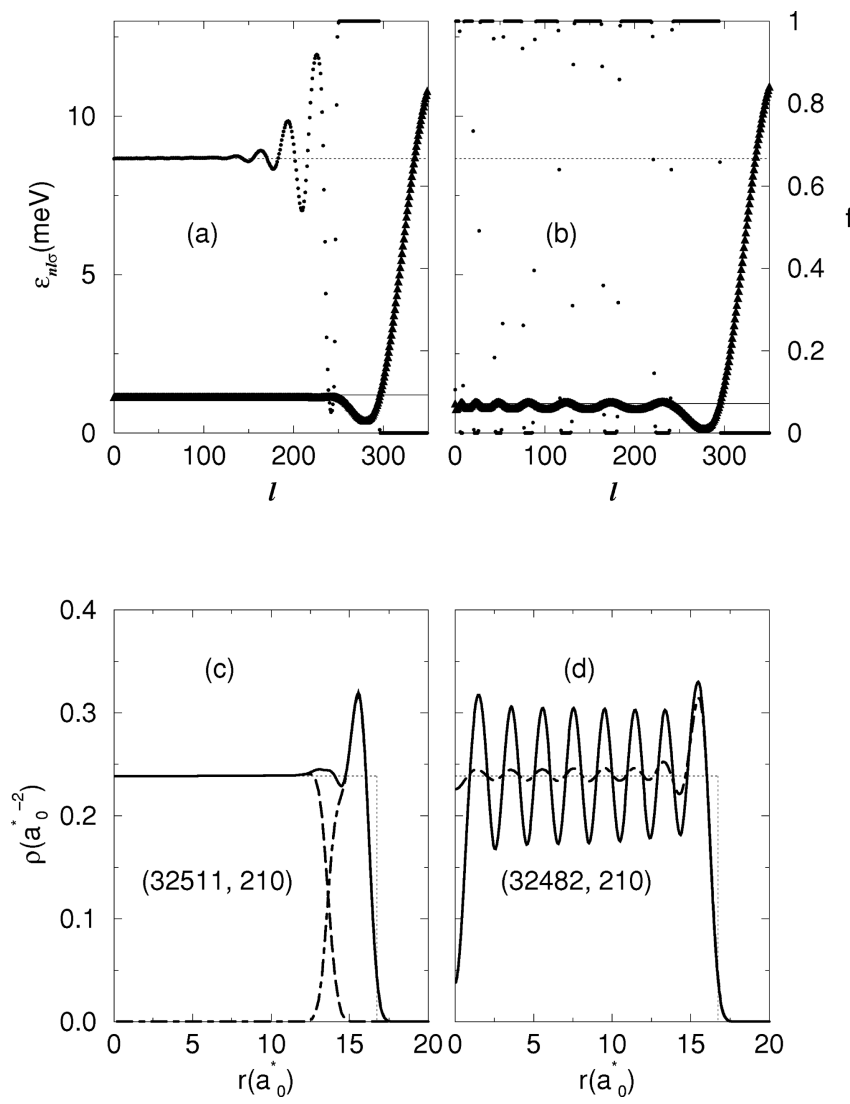

FIG. 8. Top panels: s.p. energies (triangles, left scale), and occupation numbers (dots, right scale), as a function of orbital angular momentum $l$ for $B=15.42 \mathrm{~T}$. The horizontal solid and dotted lines represent, respectively, the electron chemical potential, and the occupation number $\frac{2}{3}$. Bottom panels: the corresponding electron density as a function of $r$, both in effective atomic units. In panel (c), the bulk density is indicated by a dashed line, and the surface density by a dot-dashed line. The dot-dashed line in panel (d) represents the electron density at $T=2 \mathrm{~K}$. Also shown are the values of $\left(L_{z}, 2 S_{z}\right)$. The right panels have been obtained dropping high- $B$ contributions to the exchange-correlation energy coming from Ref. 29.

panel (a) of Fig. 8 shows that the occupation number of the s.p. levels with $l \leqslant 210$ is quite similar, around $2 / 3$. Of course, $f_{i}$ are thermal occupation numbers, and to have a fractional value, a finite but small $T$ has to be used in the calculation $(0.05 \mathrm{~K}$ in the present case). However, it is a workable way CDFT has to accommodate noninteger filling factors in the bulk of an extended droplet. Whether the occupation numbers represent a "simple" thermal situation or come from a genuine correlation effect depends on whether correlation effects have been smeared out by an inadequate high temperature or not. We want to stress that the temperature we have used in the present calculations (50 or $100 \mathrm{mK}$ ) is much lower than the one at which most experiments on quantum dots have been carried out. It is worth recalling that within the zero-temperature KS scheme, noninteger occupation numbers may appear for s.p. $i$ states such that $\epsilon_{i}=\mu{ }^{38}$ The generalization of density-functional theory to this situation is called ensemble density-functional theory, and has been used in Ref. 23 to describe quantum dots in the fractional quantum Hall effect regime.

It is worth pointing out that confining potentials produced
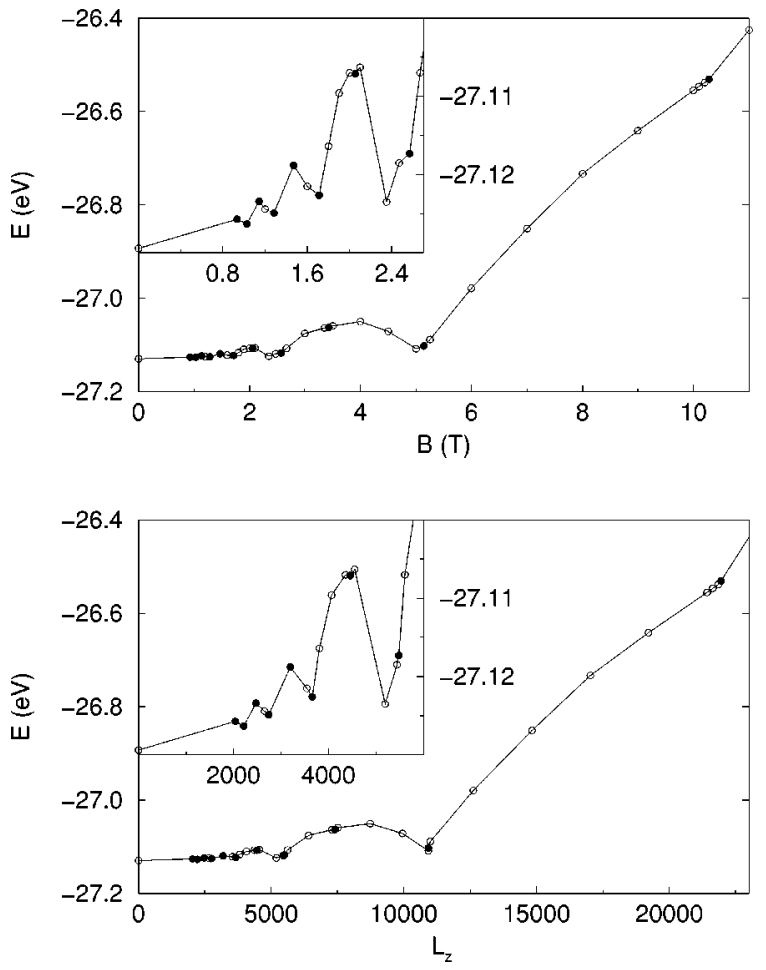

FIG. 9. Top panel: total energy as a function of $B$. The values corresponding to integer filling factors 1 to 11 are indicated by solid symbols. The line connecting the dots is to guide the eye. The inset shows the low- $B$ region, with the energy scale at the right. Bottom panel: Same as top panel but as a function of $L_{z}$.

by a jellium disk favor the existence of incompressible regions in the bulk of the dot, since there is a tendency in the electron bulk density to have a value around that of the jellium background in order to screen the Coulomb potential. This can be seen, for example, in the compressible states shown in Fig. 6. It is also clear that whenever an incompressible state appears, its bulk density is that of the neutralizing jellium; see Figs. 3 and 8. However, a physically sound exchange-correlation energy is at the very origin of the incompressible states we have found.

To substantiate our case, we have displayed in panels (b) and (d) of Fig. 8 the results obtained at $B=15.42 \mathrm{~T}$ when the exchange-correlation energy of Ref. 29 is dropped from the functional. This supports that the results shown in panels (a) and (c) of that figure are not only the trivial consequence of screening the jellium density, nor of using an inadequate high temperature, but a product of the high- $B$ correlations included in the density functional. Actually, one can see from panel (d) that density oscillations are clearly visible even at $T=2 \mathrm{~K}$.

For integer $\nu$ values, the changes are not so dramatic, and the Tanatar-Ceperly exchange-correlation energy alone yields results quite similar to those we have shown for $\nu$ $\geqslant 1$.

The $B$ dependence of the total energy $E$ is shown in the top panel of Fig. 9. Local minima in $E$ are clearly visible at or near even $\nu$ values corresponding to paramagnetic states of the droplet having "zero" total spin momentum. Local maxima are at or near odd $\nu$ values, which correspond to ferromagnetic states having large $2 S_{z}$ values, but smaller than these given by Eq. (15). The energy also presents an 


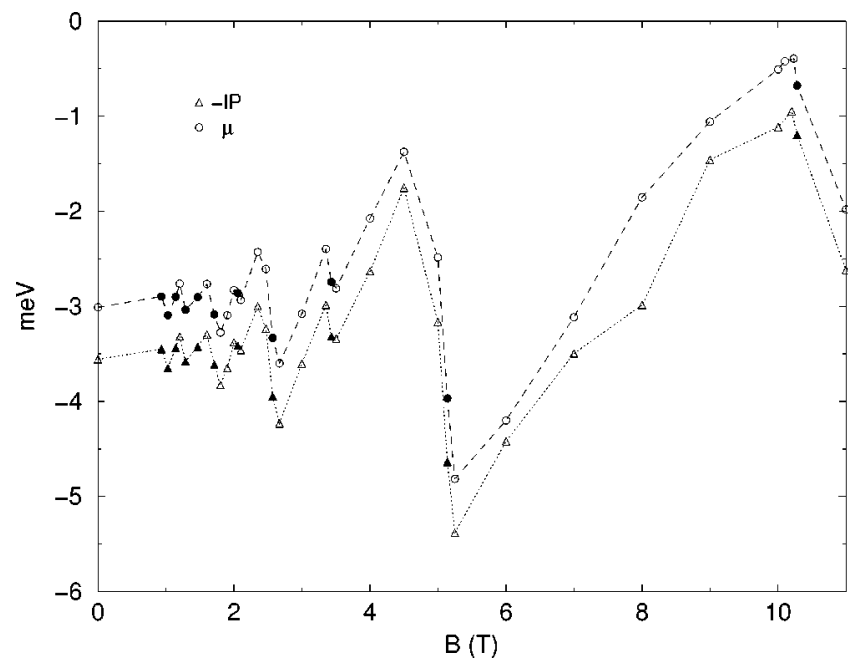

FIG. 10. Electron chemical potential (circles), and $-I_{P}$ (triangles), as a function of $B$. The lines connecting the points are to guide the eye. The points corresponding to integer filling factors 1 to 11 are indicated by solid symbols.

inflection point at the ferromagnetic $\nu=1$ state with $2 S_{z}$ $=210$. In contradistinction with the situation for small dots where only one transition from paramagnetic to ferromagnetic g.s.'s is observed, ${ }^{39}$ several smooth transitions take place below $B \sim 10 \mathrm{~T}$ for $N=210$. Also shown in that figure (bottom panel) is the dependence of $E$ with $L_{z}$, with local minima at the $L_{z}$ values corresponding to even filling factors.

The existence of several paramagnetic states plays a crucial role in keeping the energy of the droplet at around the value corresponding to $B=0$. $|E|$ decreases as $S_{z}$ increases, and vice versa. It is only from the $\nu=2$ state on that $E$ increases monotonously, as $S_{z}$ does up to reaching the full polarized value around $\nu=1$.

The electron chemical potential and the ionization potential $I_{P}=E_{N-1}-E_{N}$ (Ref. 40) are shown in Fig. 10. They display a "sawtooth" behavior with $B$, with a fast falling near integer $\nu$ values. The corresponding $B$ values or, equivalently, the corresponding $L_{z}$ values, are a kind of "magic numbers" for which the dot is particularly stable. It is worth noticing that the large size of this dot is the reason why $-I_{P}$ and $\mu$ are so similar, and why the oscillations in $I_{P}$ are rather wide, extending over several tesla and giving them the possibility of being experimentally observed more easily than in small dots. ${ }^{7,8}$

We have not attempted a systematic study of the addition energies $E_{\mathrm{ad}}$, but have obtained them for $B=4$ and $7 \mathrm{~T}$. To this end, we have calculated $E(209)$, keeping $\rho_{b}$ constant and adjusting $R$ so as to have $N_{+}=209$. Subtracting $E$ (209) from $E(210)$ yields $E_{a d} \sim 192 \mathrm{meV}$ and $190 \mathrm{meV}$, respectively. These values agree well with those obtained with the schematic model of Ref. 13, which are 215 and $213 \mathrm{meV}$, respectively.

\section{FAR-INFRARED EDGE MODES}

In this section we present the results obtained for the farinfrared edge modes of the $N=210$ dot and compare them with the experimental results of Ref. 22. The method we have used is thoroughly described in Ref. 21. It is based on a

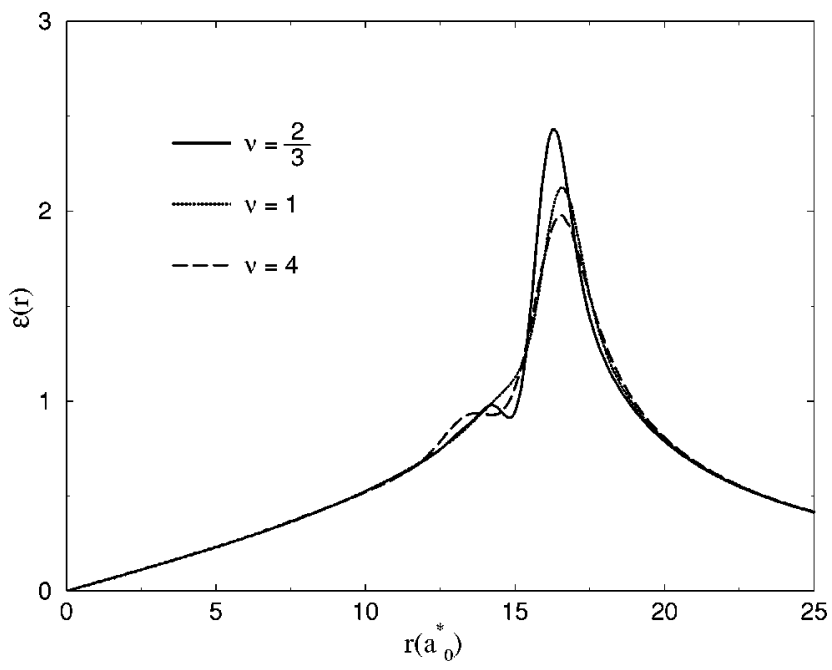

FIG. 11. Electron electric field $\mathcal{E}(r)$ in effective atomic units as a function of $r$ for filling factors $\nu=\frac{2}{3}, 1$, and 4 .

variational solution of the equation of motion obeyed by the excitation operator that generates the modes, much along the method originally proposed by Feynman to describe density excitations of superfluid ${ }^{4} \mathrm{He}^{41}$

The expressions to obtain the frequencies of edge modes of different multipolarity $L$ are rather cumbersome, but only make use of one-body densities associated with the ground state of the system. In the dipole $L=1$ case, the one for which experimental results have been unambiguously obtained, we worked out a simple expression for the $\omega_{ \pm 1}$ frequency: ${ }^{21}$

$$
\omega_{ \pm 1}=\sqrt{\frac{\omega_{c}^{2}}{4}+\frac{1}{2 N} \int d \vec{r} \Delta V_{+}(r) \rho(r)} \pm \frac{\omega_{c}}{2} .
$$

It is easy to further elaborate on this equation and write the dipole frequency as a function of the electric field $\mathcal{E}(r)$ created by the electrons at the edge of the dot. We obtain

$$
\omega_{ \pm 1}=\sqrt{\frac{\omega_{c}^{2}}{4}+\frac{\mathcal{E}(R)}{R}} \pm \frac{\omega_{c}}{2} .
$$

We have drawn $\mathcal{E}(r)$ in Fig. 11 for the $B$ values corresponding to $\nu=\frac{2}{3}, 1$, and 4 . As has been previously obtained, ${ }^{42}$ it is sharply peaked at the dot edge. The shoulders displayed by $\mathcal{E}(r)$ near the edge reflect the complex structure of the droplet density in that region.

We show in Fig. 12 the frequencies corresponding to the $L= \pm 1, \pm 2$, and \pm 3 modes, together with the experimental points of Ref. 22. We want to stress that there are no adjustable parameters in the calculation. From the figure, one sees that the agreement between theoretical and experimental spectra is good. In particular, the $L= \pm 1$ branches are well reproduced up to a high value of $B$. The interference between the $\omega_{-3}$ and $\omega_{+1}$, which Shikin et al. ${ }^{43}$ suggest causes the level repulsion experimentally found, is at the right place and has the correct amplitude. In our calculation, the quadrupole branches fit some experimental points. However, it is not yet clear whether these points correspond to quadrupole 


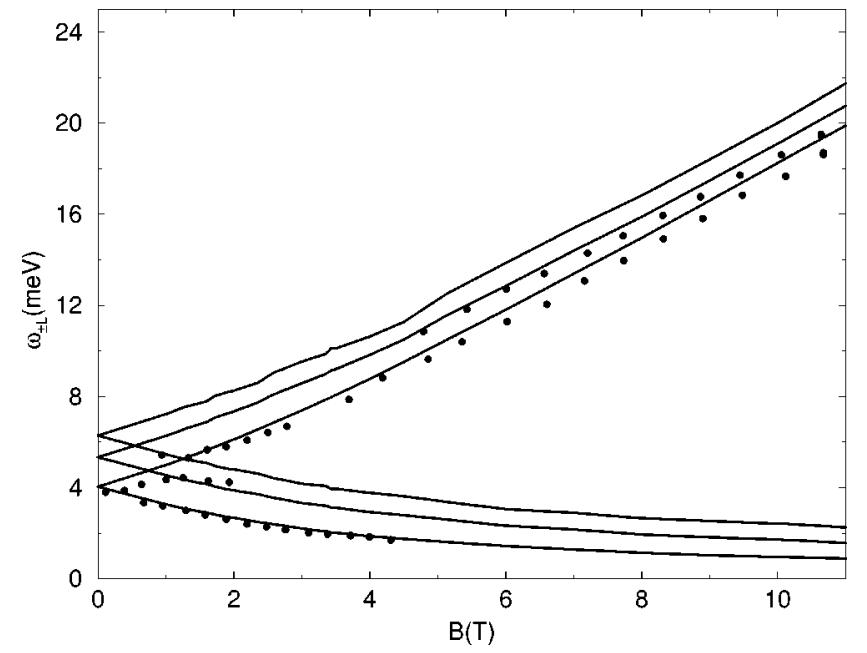

FIG. 12. Edge mode frequencies as a function of $B$. At $B \sim 0$, from top to bottom the curves correspond to $L= \pm 3, \pm 2$, and \pm 1 . The experimental dots are from Ref. 22.

excitations,${ }^{44}$ or are the result of a fragmentation of the dipole spectrum, as some calculations in small dots might indicate. ${ }^{45}$

In a recent work, ${ }^{9}$ it has been pointed out that incompressible strips at the edge of quantum dots could be detected by far-infrared spectroscopy when the confining potential is nonparabolic. In particular, the low-frequency dipole branch should be especially sensitive to the shape of the dot edge and, hence, exhibits oscillations as $B$ is varied. Equation (19) shows that these oscillations appear if the electric field at the edge of the dot has an oscillatory behavior with $B$, and are absent otherwise. For example, when the confining potential $V_{+}(r)$ is parabolic, Eqs. (18) and (19) yield nonoscillatory dipole frequencies, as $\mathcal{E} / R$ is a constant. This is easily seen from Eq. (18). In particular, that constant is $\omega_{0}^{2}=\pi \rho_{b} / R$ if the parabolic approximation of $V_{+}$in the $r / R \ll 1$ limit, $V_{+}(r)=\frac{1}{2} \omega_{0}^{2} r^{2}$, is justified.

We have analyzed the negative dispersion branch of the dipole mode represented in Fig. 12, plotting the ratio $\omega_{-} / \omega_{-}^{c l}$ as a function of $B$, where $\omega_{-}^{c l}$ is given by Eq. (19) taking for $\mathcal{E}(R)$ its value at $B=0$. The results are shown in Fig. 13. One can see that the oscillations in $\omega_{-}$are indeed filling factor related, and as indicated in Ref. 9, the minima correspond to half-filled Landau levels (odd $\nu$ ), and the maxima to fully occupied Landau levels (even $\nu$ ). Filling factor $\nu=1$, not attained in Ref. 9, is an exception as it constitutes in a sense a filled level by itself. The dipole positive dispersion branch also presents oscillations, but they are an order of magnitude smaller than these of the $\omega_{-}$branch.

Apart from the $\nu=1$ and 2 cases, the amplitude of the oscillations we find is smaller than in Ref. 9, but one should have in mind that different confining potentials are used. It is also worth noticing that in the present calculation, the oscillations in the dipole frequency are not due to the existence of integer filling factor strips at the surface of the dot, which as we have indicated before, do not appear here because of the sharp jellium density we are using. Rather, they arise due to the appearance of integer filling factor regions in the bulk of the dot.

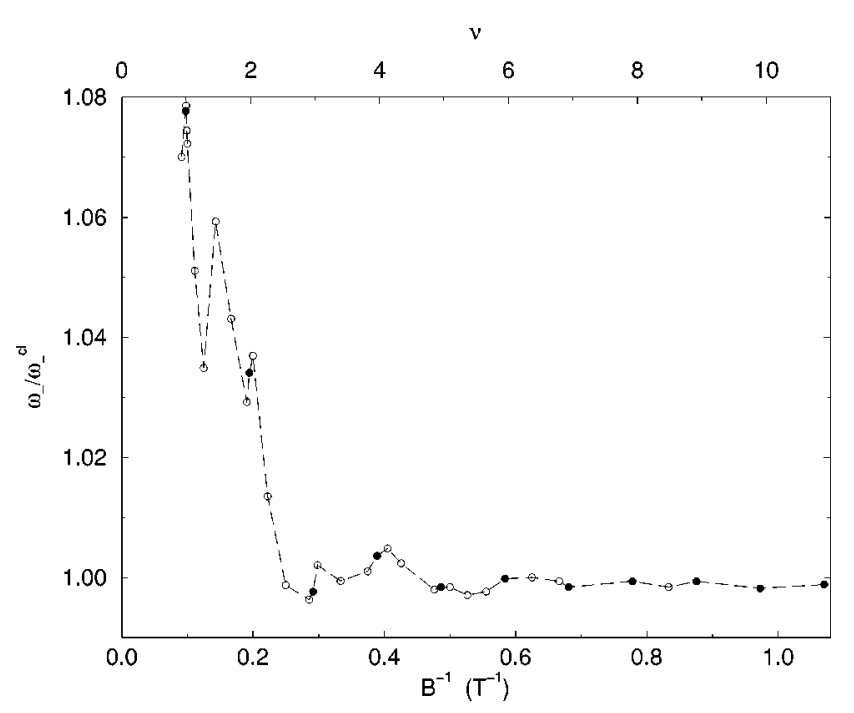

FIG. 13. $\omega_{-} / \omega_{-}^{c l}$ as a function of $1 / B$ (lower scale) and $\nu$ (upper scale). The solid symbols correspond to integer filling factors 1-11.

\section{SUMMARY}

In this work we have used current-density-functional theory to describe the ground state and multipole spectrum of a quantum dot made of 210 electrons, confined in a disk geometry and submitted to weak and intense magnetic fields as well. The sharp jellium confining potential does not allow the system to exhibit incompressible states at the edge, but its large size permits these states to develop in the bulk. We have been able to identify such incompressible states with filling factors from one to ten, and many other compressible states in between.

At $B=15.42 \mathrm{~T}$, a $\nu=\frac{2}{3}$ incompressible state appears with two-thirds of the electrons of the dot in the bulk region, and one-third in the edge region. The occupation number of the single-particle states in the bulk region is around $2 / 3$, and near the edge they rise to unity. These features have also been found in Ref. 23 when the confining potential is supplied by a positive jellium background, and have been interpreted there as the formation of a composite edge in the system.

The finite temperature version of CDFT permits one to solve a technical problem when the density of s.p. states is large and it is impracticable to solve the zero-temperature KS equations with s.p. occupation numbers 0 or 1. Interestingly, at low enough temperatures it permits one to obtain the electron density and other closely related characteristics of highly correlated states that cannot be described even approximately in terms of integer occupation numbers, provided of course, these correlations are built in the functional. This is precisely the present case, and also that of Refs. 4 and 23.

We predict values of the orbital and spin angular momentum that agree well with the ones pertaining to Landau and Laughlin states. States with integer filling factors are rather robust against changes in $B$. In particular, the study of the total energy, ionization and electron chemical potentials as a function of $B$ has shown that even $\nu, S_{z} \sim 0$ paramagnetic states are especially stable, corresponding to local minima of the total energy, and with large ionization and electron chemical potentials. Odd $\nu$, ferromagnetic states with large 
$S_{z}$ values correspond to local energy maxima, apart from the particular fully spin polarized $\nu=1$ state, and also have comparatively small ionization and electron chemical potentials.

We have stressed the important role played by the exchange-correlation term of the current density functional in the quantitative description of these effects on the one hand, and the need to perform the calculations at small temperatures to disclose fine features of incompressible states, on the other hand.

Finally, we have studied the multipole spectrum of the dot. Good agreement with the experimental data has been found. For the dipole mode, we have been able to associate the oscillations with $B$ of the dipole frequencies around the classical value, with the oscillations with $B$ of the electron electric field at the edge of the dot. These oscillations are filling factor related and in our case are associated to the formation of bulk incompressible disks, and in the case of Ref. 9 to the formation of edge incompressible strips.

\section{ACKNOWLEDGMENTS}

It is a pleasure to thank Nuria Barberán for useful discussions. This work has been performed under Grant Nos. PB95-1249 and PB95-0492 from CICYT, and SAB95-0388 from DGID, Spain, and GRQ94-1022 from Generalitat of Catalunya. A.E. acknowledges support from the Dirección General de Enseñanza Superior (Spain).
*Permanent address: Dipartimento di Fisica, Università di Trento, 38050 Povo, Italy.

${ }^{1}$ A. M. Chang, Solid State Commun. 74, 871 (1990).

${ }^{2}$ C. W. J. Bennakker, Phys. Rev. Lett. 64, 216 (1990).

${ }^{3}$ D. B. Chklovskii, B. I. Shklovskii, and L. I. Glazman, Phys. Rev. B 46, 4026 (1992).

${ }^{4}$ M. Ferconi, M. R. Geller, and G. Vignale, Phys. Rev. B 52, 16 357 (1995)

${ }^{5}$ P. A. Maksym, Physica B 184, 385 (1993).

${ }^{6}$ C. de C. Chamon and X. G. Wen, Phys. Rev. B 49, 8227 (1994).

${ }^{7}$ P. L. McEuen, E.B. Foxman, U. Meirav, M. A. Kastner, Y. Meir, N. S. Wingreen, and S. J. Wind, Phys. Rev. Lett. 66, 1926 (1991).

${ }^{8}$ R. C. Ashoori, H. L. Stormer, J. S. Weiner, L. N. Pfeiffer, S. J. Pearton, K. W. Baldwin, and K. W. West, Phys. Rev. Lett. 68, 3088 (1992); R. C. Ashoori, H. L. Stormer, J. S. Weiner, L. N. Pfeiffer, K. W. Baldwin, and K. W. West, ibid. 71, 613 (1993).

${ }^{9}$ K. Bollweg, T. Kurth, D. Heitmann, V. Gudmundsson, E. Vasiliadou, P. Grambow, and K. Eberl, Phys. Rev. Lett. 76, 2774 (1996).

${ }^{10}$ P. A. Maksym and T. Chakraborty, Phys. Rev. Lett. 65, 108 (1990); Phys. Rev. B 45, 1947 (1992).

${ }^{11}$ D. Pfannkuche, V. Gudmundsson, and P. A. Maksym, Phys. Rev. B 47, 2244 (1993).

${ }^{12}$ P. Hawrylak and D. Pfannkuche, Phys. Rev. Lett. 70, 485 (1993).

${ }^{13}$ S.-R. E. Yang, A. H. MacDonald, and M. D. Johnson, Phys. Rev. Lett. 71, 3194 (1993).

${ }^{14}$ E. H. Lieb, J. Ph. Solovej, and J. Yngvason, Phys. Rev. B 51, 10 646 (1995).

${ }^{15}$ C. W. J. Beenakker, H. van Houten, and A. A. M. Staring, Phys. Rev. B 44, 1657 (1991).

${ }^{16}$ P. L. McEuen, E. B. Foxman, J. Kinaret, U. Meirav, M. A. Kastner, N. S. Wingreen, and S. J. Wind, Phys. Rev. B 45, 11419 (1992).

${ }^{17}$ V. Gudmundsson and J. J. Palacios, Phys. Rev. B 52, 11266 (1995).

${ }^{18}$ M. M. Fogler, E. I. Levin, and B. I. Shklovskii, Phys. Rev. B 49, 13767 (1994).

${ }^{19}$ G. Vignale and M. Rasolt, Phys. Rev. Lett. 59, 2360 (1987); Phys. Rev. B 37, 10685 (1988)

${ }^{20}$ M. Ferconi and G. Vignale, Phys. Rev. B 50, 14722 (1994).

${ }^{21}$ E. Lipparini, N. Barberán, M. Barranco, M. Pi, and Ll. Serra, Phys. Rev. B 56, 12375 (1997)

${ }^{22}$ T. Demel, D. Heitmann, P. Grambow, and K. Ploog, Phys. Rev. Lett. 64, 788 (1990).
${ }^{23}$ O. Heinonen, M. I. Lubin, and M. D. Johnson, Phys. Rev. Lett. 75, 4110 (1995); M. I. Lubin, O. Heinonen, and M. D. Johnson, Phys. Rev. B 56, 10373 (1997).

${ }^{24}$ It should be noted that in Refs. 20,21, it was used an effective $\mu_{B}^{*}$ defined taking $m$ instead of $m_{e}$. We have checked that the energies of the edge modes discussed in Ref. 21 are essentially unaffected by the misuse of $\mu_{B}^{*}$ in the Zeeman splitting.

${ }^{25}$ D. A. Broido, K. Kempa, and P. Bakshi, Phys. Rev. B 42, 11400 (1990).

${ }^{26}$ D. B. Chklovskii, Phys. Rev. B 51, 9895 (1995).

${ }^{27}$ A. H. MacDonald, S. R. E. Yang, and M. D. Johnson, Aust. J. Phys. 46, 345 (1993).

${ }^{28}$ B. Tanatar and D. M. Ceperley, Phys. Rev. B 39, 5005 (1989).

${ }^{29}$ D. Levesque, J. J. Weis, and A. H. MacDonald, Phys. Rev. B 30, 1056 (1984).

${ }^{30}$ O. Klein, C. de C. Chamon, D. Tang, D. M. Abusch-Magder, U. Meirav, X.-G. Wen, M. A. Kastner, and S. J. Wind, Phys. Rev. Lett. 74, 785 (1995).

${ }^{31}$ M. Ferconi and G. Vignale, Phys. Rev. B 56, 12108 (1997).

${ }^{32}$ M. Brack and P. Quentin, Phys. Lett. 52B, 159 (1974).

${ }^{33}$ M. Brack, O. Genzken, and K. Hansen, Z. Phys. D 21, 65 (1991).

${ }^{34}$ S. Weisgerber and P.-G. Reinhard, Z. Phys. D 23, 275 (1992).

${ }^{35}$ R. B. Laughlin, Phys. Rev. Lett. 50, 1395 (1983).

${ }^{36}$ S. Mitra and A. H. MacDonald, Phys. Rev. B 48, 2005 (1993).

${ }^{37}$ R. Ferrari and N. Datta, Bull. Am. Phys. Soc. 37, 164 (1992).

${ }^{38}$ R. M. Dreizler and E. K. U. Gross, Density Functional Theory (Springer-Verlag, Berlin, 1990).

${ }^{39}$ J. J. Palacios, L. Martín-Moreno, G. Chiappe, E. Louis, and C. Tejedor, Phys. Rev. B 50, 5760 (1994).

${ }^{40}$ To avoid any misunderstanding, we remark that to obtain the electron ionization potential we have not changed at all the positive background, but simply have subtracted one electron and have carried out the minimization for the $N=209$, positively charged dot.

${ }^{41}$ R.P. Feynmann, Statistical Mechanics (Benjamin, Reading, MA, 1972).

${ }^{42}$ M. Stone, H. W. Wyld, and R. L. Schult, Phys. Rev. B 45, 14156 (1992).

${ }^{43}$ V. Shikin, S. Nazin, D. Heitmann, and T. Demel, Phys. Rev. B 43, 11903 (1991).

${ }^{44}$ M. Wagner, A. V. Chaplik, U. Merkt, Phys. Rev. B 51, 13817 (1995).

${ }^{45}$ V. Gudmundsson and R. R. Gerhardts, Phys. Rev. B 43, 12098 (1991). 